\title{
The Enthalpies of Mixing of Liquid Ni-Sn-Zn Alloys
}

\author{
Yu. Plevachuk, A. Yakymovych, S. Fürtauer, H. Ipser, and H. Flandorfer
}

(Submitted December 12, 2013; in revised form January 31, 2014; published online February 22, 2014)

\begin{abstract}
The partial and integral enthalpies of mixing of liquid ternary Ni-Sn-Zn alloys were determined. The system was investigated along two sections $x_{\mathrm{Ni}} / x_{\mathrm{Sn}} \approx 1: 9, x_{\mathrm{Ni}} / x_{\mathrm{Sn}} \approx 1: 6$ at $1073 \mathrm{~K}$ and along two sections $x_{\mathrm{Sn}} / x_{\mathrm{Zn}} \approx 9: 1, x_{\mathrm{Sn}} / x_{\mathrm{Zn}} \approx 4: 1$ at $873 \mathrm{~K}$. The integral enthalpy of mixing at each temperature is described using the Redlich-Kister-Muggianu model for substitutional ternary solutions. In addition, the experimental results were compared with data calculated according to the Toop extrapolation model. The minimum integral enthalpy of approx. $-20000 \mathrm{~J} \mathrm{~mol}^{-1}$ corresponds to the minimum in the constituent binary Ni-Sn system, the maximum of approx. $3000 \mathrm{~J} \mathrm{~mol}^{-1}$ is equal to the maximum in the binary $\mathrm{Sn}-\mathrm{Zn}$ system.
\end{abstract}

Keywords calorimetry, enthalpy of mixing, metallic alloys, ternary

\section{Introduction}

Low temperature soldering is one of the key technologies for the production of electronics devices. Recently, several types of new lead-free Sn-based solders have attracted the attention of the electronics industry. ${ }^{[1-4]}$ Eutectic or near eutectic Sn-Zn alloys, which have been recognized as possible solder candidates due to their low melting temperatures and low costs, are among them.

The melting point of the eutectic $\mathrm{Sn}_{85.1} \mathrm{Zn}_{14.9}$ solder is $472 \mathrm{~K}$, which is close to that of the conventional $\mathrm{Sn}-\mathrm{Pb}$ eutectic alloy $(456 \mathrm{~K})$ but lower than those of other Sn-based eutectic alloys that are already used in soldering, i.e. $\mathrm{Sn}-\mathrm{Cu}(500 \mathrm{~K}), \mathrm{Sn}-\mathrm{Ag}$ (494 K) or $\mathrm{Sn}-\mathrm{Ag}-\mathrm{Cu}$ (490 K). While the $\mathrm{Sn}-\mathrm{Zn}$ eutectic alloy has excellent properties as a low temperature solder, it has also some drawbacks. Damage by heat exposure and corrosion in humidity, inferior wettability, easy oxidation and micro-void formation have been encountered to limit the practical use of this solder. ${ }^{[5]}$

It is known that the poor oxidation resistance of the $\mathrm{Sn}-\mathrm{Zn}$ eutectic alloy is due to zinc oxidation which occurs both in the primary crystallization of $(\mathrm{Zn})$ and the eutectic phase. If the amount of the ( $\mathrm{Zn})$ phase in the Sn-Zn eutectic alloy can be reduced or fixed by formation of intermetallic compounds, it is expected that the oxidation resistance can be improved. ${ }^{[6]}$ Therefore, much research was focused on the addition of alloying elements, such as $\mathrm{Cu}, \mathrm{Ni}, \mathrm{Ag}, \mathrm{Sb}$, or $\mathrm{Bi}$,. Among them, $\mathrm{Ni}$ has been considered as a suitable

Yu. Plevachuk, Department of Metal Physics, Ivan Franko National University, Kyrylo and Mephodiy Str. 8, Lviv 79005, Ukraine; A. Yakymovych, S. Fürtauer, H. Ipser, and H. Flandorfer, Department of Inorganic Chemistry (Materials Chemistry), University of Vienna, Währinger Str. 42, 1090 Wien, Austria. Contact e-mail: andriy.yakymovych@univie.ac.at. alloying element in lead-free solders due to the formation of stable Ni-Zn binary phases as well as by improving the wettability. ${ }^{[7]}$ Furthermore, the addition of Ni effectively enhances the formation of additional ternary intermetallic compounds which can improve the mechanical properties. ${ }^{[8]}$

The development of lead-free solders requires a clear and thorough understanding of their structural and thermodynamic properties. The increasing influence of computational modelling in all technological processes generates an increased demand for accurate thermodynamic information for the materials systems involved, which are used as fundamental inputs for any model. The solidification process of a liquid alloy has a profound impact on the structure and properties of the solid material. Therefore, knowledge of the basic properties of the molten alloys prior to solidification becomes very important for the development of materials with predetermined characteristics.

In this work the enthalpy of mixing of Sn-based liquid Ni-Sn-Zn alloys was investigated at 873 and $1073 \mathrm{~K}$. The data obtained are useful for modelling of interatomic interactions of the components as well as for a thermodynamic assessment of the Ni-Sn-Zn system. The experimental data were fitted on the basis of an extended RedlichKister-Muggianu model ${ }^{[9]}$ and compared to data calculated according to the Toop extrapolation model. ${ }^{[10]}$

\section{Literature Survey}

\subsection{The Sn-Zn Binary System}

Thermodynamic properties of liquid $\mathrm{Sn}-\mathrm{Zn}$ alloys have been investigated repeatedly. ${ }^{[11-20]}$ The authors used different methods to determine the enthalpy of mixing: calorimetric investigations were carried out in Ref 11-15, the authors of Ref 16-19 used the emf method, and quantitative thermal analysis was used in Ref 20. An endothermic behavior of $\Delta_{\mathrm{Mix}} H$ has been revealed over the whole concentration region with a maximum point at about 65 at.\% Zn; only Kleppa reported a temperature dependence of the integral enthalpy of mixing. ${ }^{[11]}$ A critical review of the experimental enthalpy of mixing data was published by Lee. ${ }^{[21]}$ 


\subsection{The Ni-Sn Binary System}

The enthalpy of mixing of liquid Ni-Sn alloys was investigated experimentally in Ref 22-24. According to Haddad et al., ${ }^{[22]}$ the enthalpy of mixing does not depend on temperature between 867 and $1579 \mathrm{~K}$ while such a dependence was reported in the temperature range $1660-1775 \mathrm{~K}$ by Lück et al. ${ }^{[23]}$

A strong temperature dependence of the limiting partial enthalpy of mixing for Ni in Sn was observed by Flandorfer et al. ${ }^{[2]}$ The authors concluded also a certain temperature dependence of the integral enthalpy of mixing in the liquid state near the liquidus curve. Several thermodynamic assessments including phase diagram calculations, based on experimental data, were carried out in the past. ${ }^{[25-27]}$

\subsection{The Ni-Zn Binary System}

Experimental data on the thermodynamic properties of liquid $\mathrm{Ni}-\mathrm{Zn}$ alloys are scarce in the literature ${ }^{[28,29]}$; all investigations point to an exothermic mixing behavior. The minimum point, shifted to the $\mathrm{Zn}$-reach side from the equiatomic concentration, can be explained by the existence of short-range order in the liquid corresponding to the rather stable $\gamma$-phase in the solid state. All thermodynamic optimizations were based on the same experimental data. ${ }^{[30-33]}$

\subsection{The Ni-Sn-Zn Ternary System}

No calorimetric data for liquid Ni-Sn-Zn alloys have been reported up to now. However, Gandova et al. ${ }^{[36]}$ attempted an extrapolation from binary thermodynamic data to obtain Gibbs energy values for ternary liquid alloys, using different geometrical models as well as the CALculation of PHAse Diagrams (CALPHAD) method. Various groups of authors reported partial ternary phase diagrams, especially isothermal sections at different temperatures. ${ }^{[34,35,37,41,42]}$

\section{Experimental Procedure}

A Calvet-type microcalorimeter HTMC-1000 (Setaram, Lyon, France), equipped with an automatic drop device for up to 30 drops, was used for the enthalpy of mixing measurements. ${ }^{[38]}$ Control and data evaluation was done with Lab View and HiQ. All measurements were performed under Ar flow (approx. $30 \mathrm{~cm}^{3} / \mathrm{min}$ ) in graphite crucibles. The microcalorimeter was calibrated at the end of each measurement series by five additions (approx. $40 \mathrm{mg}$ each) of standard $\alpha-\mathrm{Al}_{2} \mathrm{O}_{3}$ supplied by the National Institute of Standards and Technology (NIST, Gaithersburg, MD, USA). The interval between individual drops was $40 \mathrm{~min}$, the acquisition interval of the heat flow was about $0.5 \mathrm{~s}$. Two thermopiles with more than 200 thermocouples of Pt/Pt-10Rh were used for the determination of the sample temperature $\left(T_{\mathrm{M}}\right)$ in the furnace and of the corresponding heat effect for each drop. The measured enthalpy $\Delta H_{\text {Signal }}$ (integrated heat flow at constant pressure) is given by

$\Delta H_{\text {Signal }}=n_{i}\left(H_{\mathrm{m}, i, T_{\mathrm{M}}}-H_{\mathrm{m}, i, T_{\mathrm{D}}}\right)+\Delta H_{\text {Reaction }}$,

where $n_{\mathrm{i}}$ is the number of moles of the added sample, $H_{\mathrm{m}}$ denotes molar enthalpies, and $T_{\mathrm{D}}$ is the drop temperature (room temperature). The molar enthalpy difference $\left(H_{\mathrm{m}, i, T_{\mathrm{M}}}-H_{\mathrm{m}, i, T_{\mathrm{D}}}\right)$ was calculated using the SGTE data for pure elements. ${ }^{[39]}$ Because of the rather small masses added, the partial enthalpies can be given directly as

$\Delta \overline{H_{i}}=\frac{\Delta H_{\text {Reaction }}}{n_{i}}$,

The integral enthalpy of mixing was calculated by summarizing the respective reaction enthalpies and dividing by the total molar amount of substance. The respective binary starting value for each section in the ternary system was calculated from the binary literature data ${ }^{[24,32,40]}$ using the interaction parameters listed in Table 3.

The enthalpy of mixing for ternary liquid Ni-Sn-Zn alloys was determined along two sections at $873 \mathrm{~K}$ where pure $\mathrm{Ni}$ was dropped into liquid $\mathrm{Sn}_{1-x} \mathrm{Zn}_{x}$ alloys $(x=0.09$ and 0.18 ) as well as along two section at $1073 \mathrm{~K}$ where pieces of pure $\mathrm{Zn}$ were dropped into liquid $\mathrm{Ni}_{x} \mathrm{Sn}_{1-x}$ alloys $(x=0.10$ and 0.15 ) (Fig. 1). A lower temperature of $873 \mathrm{~K}$ was chosen for sections $\mathrm{C}$ and $\mathrm{D}$ to avoid excessive $\mathrm{Zn}$ losses by evaporation. For sections A and B a higher temperature of $1073 \mathrm{~K}$ was chosen in order to cover a larger liquid range.

Random errors as well as systematic errors of calorimetry depend on different factors, such as construction of the calorimeter, calibration procedure, signal integration or "chemical errors", e.g. incomplete reactions or impurities. Considering many calibration measurements done by dropping NIST standard sapphire, the standard deviation can be estimated to be less than $\pm 1 \%$. The systematic errors are mainly caused by parasitic heat flows, base line problems at signal integration and dropping and mixing problems. One can estimate that the random error of the measured enthalpy is about $\pm 150 \mathrm{~J}$.

All experimental details, i.e. starting amounts, added amounts and resulting heat effects as well as the obtained enthalpy of mixing values are collected in Tables 1 and 2 . Figure 2, 3, 4, and 5 show the changes of $\Delta_{\text {Mix }} H$ versus concentration.

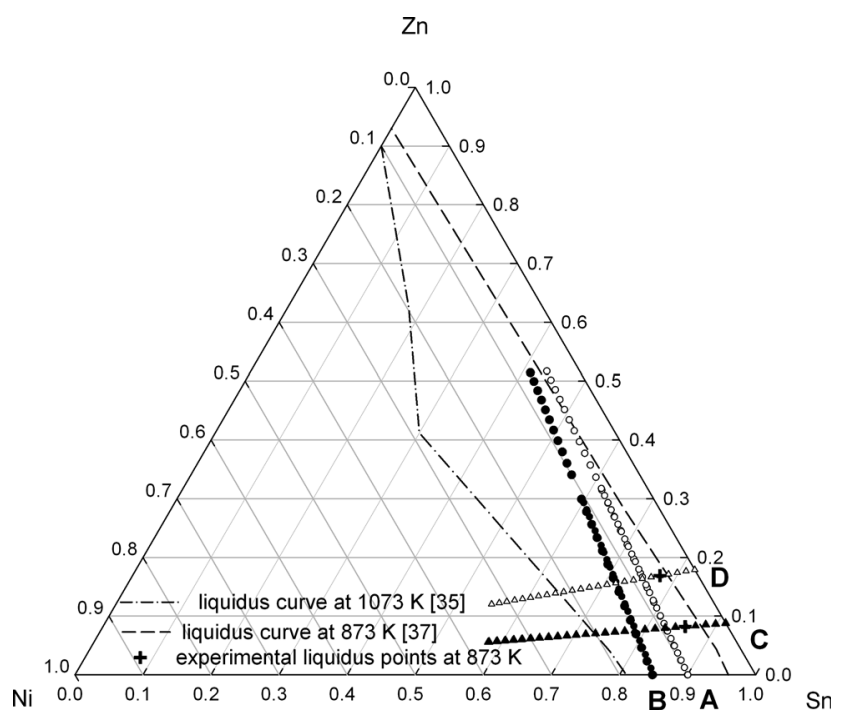

Fig. 1 Investigated sections and alloy compositions in the ternary Ni-Sn-Zn system (A and B at $1073 \mathrm{~K}$; C and D at $873 \mathrm{~K}$ ) 
Table 1 Partial and integral enthalpies of mixing of Ni-Sn-Zn alloys, $1073 \mathrm{~K}$; standard states: pure liquid metals

\begin{tabular}{|c|c|c|c|c|c|c|}
\hline \multirow{2}{*}{$\begin{array}{l}\text { Dropped mole } \\
n_{i}, 10^{-3} \mathrm{~mol}\end{array}$} & \multirow{2}{*}{$\begin{array}{c}\text { Drop enthalpy } \\
\Delta H_{\text {Signal }}, \mathrm{J} \mathrm{mol}^{-1}\end{array}$} & \multicolumn{2}{|c|}{ Partial enthalpy } & \multicolumn{3}{|c|}{ Integral enthalpy(a) } \\
\hline & & $x_{i}(\mathbf{b})$ & $\Delta \overline{\boldsymbol{H}_{i}}, \mathbf{J} \mathrm{mol}^{-1}$ & $x_{\mathbf{Z n}}$ & $x_{\text {Sn }}$ & $\Delta_{\mathrm{Mix}} \boldsymbol{H}, \mathbf{J} \mathrm{mol}^{-1}$ \\
\hline \multicolumn{7}{|c|}{ Sect. $A: x_{\mathrm{Ni}} / x_{\mathrm{Sn}} \approx 1: 9 ; i=\mathrm{Zn} ;$ starting amounts: $n_{\mathrm{Ni}}=1.8795 \times 10^{-3} \mathrm{~mol} ; n_{\mathrm{Sn}}=16.9010 \times 10^{-3} \mathrm{~mol}$} \\
\hline 0 & $\ldots$ & 0 & $\ldots$ & 0 & 0.8999 & -4575 \\
\hline 0.4742 & 35463 & 0.0123 & 5404 & 0.0246 & 0.8778 & -4329 \\
\hline 0.9874 & 35289 & 0.0373 & 5230 & 0.0499 & 0.8550 & -4081 \\
\hline 1.5146 & 34981 & 0.0623 & 4922 & 0.0746 & 0.8328 & -3847 \\
\hline 2.0829 & 34621 & 0.0872 & 4562 & 0.0998 & 0.8101 & -3618 \\
\hline 2.6552 & 34988 & 0.1119 & 4929 & 0.1239 & 0.7884 & -3390 \\
\hline 3.2758 & 35026 & 0.1362 & 4967 & 0.1485 & 0.7663 & -3155 \\
\hline 3.9210 & 34624 & 0.1606 & 4564 & 0.1727 & 0.7445 & -2935 \\
\hline 4.6136 & 35104 & 0.1850 & 5044 & 0.1972 & 0.7224 & -2699 \\
\hline 5.3408 & 34808 & 0.2093 & 4749 & 0.2214 & 0.7007 & -2474 \\
\hline 6.1164 & 34346 & 0.2335 & 4287 & 0.2457 & 0.6788 & -2264 \\
\hline 6.9339 & 34532 & 0.2577 & 4472 & 0.2697 & 0.6573 & -2050 \\
\hline 7.7665 & 34110 & 0.2811 & 4050 & 0.2926 & 0.6366 & -1858 \\
\hline 8.6252 & 34158 & 0.3036 & 4099 & 0.3147 & 0.6167 & -1672 \\
\hline 9.5212 & 34142 & 0.3256 & 4083 & 0.3364 & 0.5972 & -1490 \\
\hline 10.4328 & 33950 & 0.3468 & 3891 & 0.3571 & 0.5785 & -1322 \\
\hline 11.3799 & 33774 & 0.3672 & 3715 & 0.3773 & 0.5604 & -1163 \\
\hline 12.3639 & 33972 & 0.3871 & 3913 & 0.3970 & 0.5427 & -1003 \\
\hline 13.3635 & 33675 & 0.4064 & 3616 & 0.4157 & 0.5258 & -859 \\
\hline 14.4186 & 33334 & 0.4250 & 3275 & 0.4343 & 0.5091 & -728 \\
\hline 15.4831 & 33464 & 0.4431 & 3405 & 0.4519 & 0.4933 & -600 \\
\hline 16.5723 & 33105 & 0.4603 & 3046 & 0.4688 & 0.4781 & -487 \\
\hline 17.7193 & 33126 & 0.4771 & 3067 & 0.4855 & 0.4630 & -376 \\
\hline 18.8975 & 32838 & 0.4935 & 2778 & 0.5016 & 0.4486 & -277 \\
\hline 20.0849 & 32818 & 0.5092 & 2758 & 0.5168 & 0.4349 & -184 \\
\hline \multicolumn{7}{|c|}{$i=\mathrm{Zn}$; starting amounts: $n_{\mathrm{Ni}}=2.8002 \times 10^{-3} \mathrm{~mol} ; n_{\mathrm{Sn}}=25.3021 \times 10^{-3} \mathrm{~mol}$} \\
\hline 0 & $\ldots$ & 0 & $\ldots$ & 0 & 0.9004 & -4554 \\
\hline 0.3509 & 35704 & 0.0062 & 5594 & 0.0123 & 0.8893 & -4429 \\
\hline 0.7141 & 35519 & 0.0186 & 5409 & 0.0248 & 0.8780 & -4305 \\
\hline 1.0846 & 35353 & 0.0310 & 5243 & 0.0372 & 0.8669 & -4184 \\
\hline 1.4534 & 34920 & 0.0432 & 4810 & 0.0492 & 0.8561 & -4072 \\
\hline 1.8465 & 35332 & 0.0554 & 5222 & 0.0617 & 0.8448 & -3950 \\
\hline 2.2588 & 34949 & 0.0680 & 4839 & 0.0744 & 0.8334 & -3830 \\
\hline 2.6943 & 35035 & 0.0809 & 4925 & 0.0875 & 0.8216 & -3707 \\
\hline 3.1405 & 35024 & 0.0940 & 4914 & 0.1005 & 0.8099 & -3583 \\
\hline 3.6093 & 34852 & 0.1072 & 4742 & 0.1138 & 0.7979 & -3460 \\
\hline 4.0840 & 34598 & 0.1204 & 4488 & 0.1269 & 0.7861 & -3343 \\
\hline 4.5754 & 34674 & 0.1335 & 4564 & 0.1400 & 0.7743 & -3224 \\
\hline 5.0883 & 34661 & 0.1467 & 4551 & 0.1533 & 0.7623 & -3104 \\
\hline 5.6137 & 34619 & 0.1599 & 4509 & 0.1665 & 0.7504 & -2985 \\
\hline 6.1585 & 34270 & 0.1731 & 4160 & 0.1798 & 0.7385 & -2872 \\
\hline 6.7115 & 33926 & 0.1863 & 3816 & 0.1928 & 0.7268 & -2766 \\
\hline 7.2719 & 34086 & 0.1992 & 3975 & 0.2056 & 0.7153 & -2659 \\
\hline 7.8535 & 34013 & 0.2120 & 3902 & 0.2184 & 0.7037 & -2553 \\
\hline 8.4508 & 34308 & 0.2248 & 4198 & 0.2312 & 0.6922 & -2442 \\
\hline 9.0626 & 33941 & 0.2375 & 3830 & 0.2438 & 0.6808 & -2339 \\
\hline 9.6893 & 33928 & 0.2501 & 3818 & 0.2564 & 0.6695 & -2237 \\
\hline 10.3270 & 33878 & 0.2626 & 3768 & 0.2687 & 0.6584 & -2137 \\
\hline 10.9854 & 33795 & 0.2749 & 3685 & 0.2810 & 0.6473 & -2039 \\
\hline 11.6546 & 33573 & 0.2871 & 3463 & 0.2931 & 0.6364 & -1947 \\
\hline 12.3527 & 33833 & 0.2992 & 3722 & 0.3053 & 0.6254 & -1849 \\
\hline 13.0640 & 33652 & 0.3113 & 3541 & 0.3173 & 0.6146 & -1756 \\
\hline
\end{tabular}


Table 1 continued

\begin{tabular}{|c|c|c|c|c|c|c|}
\hline \multirow{2}{*}{$\begin{array}{l}\text { Dropped mole } \\
n_{i}, 10^{-3} \mathrm{~mol}\end{array}$} & \multirow{2}{*}{$\begin{array}{c}\text { Drop enthalpy } \\
\Delta H_{\text {Signal }}, \mathrm{J} \mathrm{mol}^{-1}\end{array}$} & \multicolumn{2}{|c|}{ Partial enthalpy } & \multicolumn{3}{|c|}{ Integral enthalpy(a) } \\
\hline & & $x_{i}(\mathbf{b})$ & $\Delta \overline{\boldsymbol{H}_{i}}, \mathrm{~J} \mathrm{~mol}^{-1}$ & $x_{\mathrm{Zn}}$ & $x_{\text {Sn }}$ & $\Delta_{\mathrm{Mix}} \mathrm{H}, \mathrm{J} \mathrm{mol}^{-1}$ \\
\hline \multicolumn{7}{|c|}{ Sect. $B: x_{\mathrm{Ni}} / x_{\mathrm{Sn}} \approx 1: 6 ; i=\mathrm{Zn}$; starting amounts: $n_{\mathrm{Ni}}=2.9697 \times 10^{-3} \mathrm{~mol} ; n_{\mathrm{Sn}}=16.6085 \times 10^{-3} \mathrm{~mol}$} \\
\hline 0 & $\ldots$ & 0 & $\ldots$ & 0 & 0.8483 & -6998 \\
\hline 0.4638 & 32640 & 0.0116 & 2549 & 0.0231 & 0.8287 & -6777 \\
\hline 0.9475 & 32614 & 0.0347 & 2523 & 0.0462 & 0.8092 & -6558 \\
\hline 1.4621 & 32814 & 0.0578 & 2723 & 0.0695 & 0.7894 & -6331 \\
\hline 2.0017 & 33008 & 0.0811 & 2917 & 0.0928 & 0.7696 & -6100 \\
\hline 2.5943 & 33133 & 0.1049 & 3042 & 0.1170 & 0.7491 & -5855 \\
\hline 3.2188 & 33097 & 0.1291 & 3006 & 0.1412 & 0.7285 & -5613 \\
\hline 3.8634 & 33414 & 0.1530 & 3324 & 0.1648 & 0.7085 & -5367 \\
\hline 4.5377 & 33395 & 0.1765 & 3305 & 0.1882 & 0.6887 & -5124 \\
\hline 5.2394 & 33541 & 0.1996 & 3451 & 0.2111 & 0.6692 & -4882 \\
\hline 5.9692 & 33080 & 0.2224 & 2989 & 0.2337 & 0.6501 & -4657 \\
\hline 6.7383 & 33327 & 0.2449 & 3236 & 0.2560 & 0.6311 & -4427 \\
\hline 7.5331 & 33075 & 0.2670 & 2984 & 0.2779 & 0.6126 & -4209 \\
\hline 8.3589 & 33090 & 0.2885 & 2999 & 0.2992 & 0.5945 & -3996 \\
\hline 10.1075 & 32834 & 0.3198 & 2743 & 0.3405 & 0.5595 & -3599 \\
\hline 11.0247 & 32574 & 0.3504 & 2483 & 0.3602 & 0.5427 & -3417 \\
\hline 11.9850 & 32599 & 0.3700 & 2508 & 0.3797 & 0.5262 & -3237 \\
\hline 12.9748 & 32487 & 0.3891 & 2396 & 0.3986 & 0.5102 & -3065 \\
\hline 13.9881 & 32414 & 0.4077 & 2324 & 0.4167 & 0.4948 & -2903 \\
\hline 15.0290 & 32383 & 0.4255 & 2292 & 0.4343 & 0.4799 & -2746 \\
\hline 16.0976 & 32258 & 0.4427 & 2167 & 0.4512 & 0.4655 & -2599 \\
\hline 17.2098 & 32304 & 0.4595 & 2213 & 0.4678 & 0.4515 & -2454 \\
\hline 18.3485 & 32392 & 0.4758 & 2301 & 0.4838 & 0.4379 & -2311 \\
\hline 19.5236 & 32303 & 0.4915 & 2212 & 0.4993 & 0.4248 & -2175 \\
\hline 20.7209 & 31894 & 0.5067 & 1804 & 0.5142 & 0.4121 & -2057 \\
\hline \multicolumn{7}{|c|}{$i=\mathrm{Zn}$; starting amounts: $n_{\mathrm{Co}}=4.4554 \times 10^{-3} \mathrm{~mol} ; n_{\mathrm{Sn}}=25.2700 \times 10^{-3} \mathrm{~mol}$} \\
\hline 0 & $\ldots$ & 0 & $\ldots$ & 0 & 0.8501 & -6915 \\
\hline 0.3400 & 32405 & 0.0057 & 2295 & 0.0113 & 0.8405 & -6811 \\
\hline 0.6997 & 32339 & 0.0172 & 2229 & 0.0230 & 0.8306 & -6704 \\
\hline 1.0643 & 32660 & 0.0288 & 2550 & 0.0346 & 0.8207 & -6594 \\
\hline 1.4415 & 32532 & 0.0404 & 2422 & 0.0463 & 0.8108 & -6485 \\
\hline 1.8368 & 32941 & 0.0522 & 2831 & 0.0582 & 0.8006 & -6369 \\
\hline 2.2541 & 32630 & 0.0643 & 2520 & 0.0705 & 0.7902 & -6253 \\
\hline 2.6894 & 32691 & 0.0767 & 2581 & 0.0830 & 0.7796 & -6134 \\
\hline 3.1366 & 33042 & 0.0892 & 2932 & 0.0954 & 0.7690 & -6011 \\
\hline 3.6035 & 32536 & 0.1018 & 2426 & 0.1081 & 0.7582 & -5892 \\
\hline 4.0867 & 33028 & 0.1145 & 2917 & 0.1209 & 0.7474 & -5766 \\
\hline 4.5768 & 32822 & 0.1271 & 2712 & 0.1334 & 0.7367 & -5645 \\
\hline 5.0817 & 32940 & 0.1397 & 2830 & 0.1460 & 0.7260 & -5522 \\
\hline 5.5951 & 33040 & 0.1522 & 2930 & 0.1584 & 0.7154 & -5400 \\
\hline 6.1267 & 33001 & 0.1646 & 2891 & 0.1709 & 0.7048 & -5277 \\
\hline 6.6752 & 32885 & 0.1771 & 2774 & 0.1834 & 0.6942 & -5155 \\
\hline 7.2416 & 33179 & 0.1896 & 3069 & 0.1959 & 0.6836 & -5029 \\
\hline 7.8181 & 33411 & 0.2021 & 3301 & 0.2082 & 0.6731 & -4901 \\
\hline 8.4095 & 33511 & 0.2144 & 3400 & 0.2205 & 0.6626 & -4773 \\
\hline 9.0243 & 33002 & 0.2267 & 2892 & 0.2329 & 0.6521 & -4651 \\
\hline 9.6558 & 32646 & 0.2390 & 2535 & 0.2452 & 0.6417 & -4536 \\
\hline 10.2992 & 32773 & 0.2513 & 2663 & 0.2573 & 0.6314 & -4420 \\
\hline 10.9507 & 32564 & 0.2633 & 2454 & 0.2692 & 0.6212 & -4310 \\
\hline 11.6234 & 32194 & 0.2752 & 2084 & 0.2811 & 0.6111 & -4206 \\
\hline
\end{tabular}


Table 1 continued

\begin{tabular}{|c|c|c|c|c|c|c|}
\hline \multirow{2}{*}{$\begin{array}{l}\text { Dropped mole } \\
n_{i}, 10^{-3} \mathrm{~mol}\end{array}$} & \multirow{2}{*}{$\begin{array}{c}\text { Drop enthalpy } \\
\Delta H_{\text {Signal }}, \mathrm{J} \mathrm{mol}^{-1}\end{array}$} & \multicolumn{2}{|c|}{ Partial enthalpy } & \multicolumn{3}{|c|}{ Integral enthalpy(a) } \\
\hline & & $x_{i}(\mathbf{b})$ & $\Delta \overline{\boldsymbol{H}_{i}}, \mathrm{~J} \mathrm{~mol}^{-1}$ & $x_{\mathrm{Zn}}$ & $x_{\mathrm{Sn}}$ & $\Delta_{\mathrm{Mix}} H, J \mathrm{~mol}^{-1}$ \\
\hline 12.3258 & 32702 & 0.2871 & 2592 & 0.2931 & 0.6009 & -4092 \\
\hline 13.0394 & 32600.8 & 0.2990 & 2491 & 0.3049 & 0.5909 & -3983 \\
\hline
\end{tabular}

Table 2 Partial and integral enthalpies of mixing of Ni-Sn-Zn alloys, $873 \mathrm{~K}$; standard states: pure liquid metals

\begin{tabular}{|c|c|c|c|c|c|c|}
\hline \multirow{2}{*}{$\begin{array}{l}\text { Dropped mole } \\
n_{\mathrm{i}}, 10^{-3} \mathrm{~mol}\end{array}$} & \multirow{2}{*}{$\begin{array}{c}\text { Drop enthalpy } \\
\Delta H_{\text {Signal }}, \mathrm{J} \mathrm{mol}^{-1}\end{array}$} & \multicolumn{2}{|c|}{ Partial enthalpy } & \multicolumn{3}{|c|}{ Integral enthalpy(a) } \\
\hline & & $x_{i}(\mathbf{b})$ & $\Delta \overline{\boldsymbol{H}_{i}}, \mathbf{J} \mathrm{mol}^{-1}$ & $x_{\mathrm{Ni}}$ & $x_{\mathrm{Sn}}$ & $\Delta_{\mathrm{Mix}} H, \mathrm{~J} \mathrm{~mol}^{-1}$ \\
\hline \multicolumn{7}{|c|}{ Sect. $C: x_{\mathrm{Sn}} / x_{\mathrm{Zn}} \approx 9: 1 ; i=\mathrm{Ni} ;$ starting amounts: $n_{\mathrm{Sn}}=25.2346 \times 10^{-3} \mathrm{~mol} ; n_{\mathrm{Zn}}=2.4245 \times 10^{-3} \mathrm{~mol}$} \\
\hline 0 & $\ldots$ & 0 & $\ldots$ & 0 & 0.9123 & 683 \\
\hline 0.4284 & -25748 & 0.0076 & -59962 & 0.0153 & 0.8984 & -242 \\
\hline 0.8846 & -24490 & 0.0231 & -58704 & 0.0310 & 0.8841 & -1176 \\
\hline 1.3535 & -13999 & 0.0388 & -48212 & 0.0467 & 0.8698 & -1936 \\
\hline 1.8391 & -3034 & 0.0545 & -37248 & 0.0623 & 0.8555 & -2518 \\
\hline 2.3383 & 7587 & 0.0701 & -26627 & 0.0780 & 0.8412 & -2919 \\
\hline 2.8598 & 17025 & 0.0858 & -17189 & 0.0937 & 0.8269 & -3163 \\
\hline 3.3915 & 16214 & 0.1015 & -17999 & 0.1092 & 0.8127 & -3417 \\
\hline 3.9359 & 16471 & 0.1169 & -17743 & 0.1246 & 0.7987 & -3664 \\
\hline 4.5032 & 16490 & 0.1323 & -17724 & 0.1400 & 0.7846 & -3912 \\
\hline 5.0829 & 16553 & 0.1476 & -17661 & 0.1552 & 0.7707 & -4155 \\
\hline 5.6891 & 16228 & 0.1629 & -17986 & 0.1706 & 0.7567 & -4406 \\
\hline 6.3094 & 16304 & 0.1782 & -17910 & 0.1857 & 0.7429 & -4653 \\
\hline 6.9459 & 15838 & 0.1932 & -18376 & 0.2007 & 0.7292 & -4905 \\
\hline 7.5905 & 15898 & 0.2080 & -18316 & 0.2153 & 0.7159 & -5151 \\
\hline 8.2575 & 16153 & 0.2226 & -18061 & 0.2299 & 0.7026 & -5390 \\
\hline 8.9571 & 16554 & 0.2373 & -17660 & 0.2446 & 0.6892 & -5625 \\
\hline 9.6625 & 16479 & 0.2518 & -17735 & 0.2589 & 0.6761 & -5854 \\
\hline 10.3824 & 16205 & 0.2659 & -18009 & 0.2729 & 0.6633 & -6084 \\
\hline 11.1205 & 16058 & 0.2798 & -18155 & 0.2868 & 0.6507 & -6314 \\
\hline 11.8841 & 16487 & 0.2936 & -17727 & 0.3005 & 0.6382 & -6534 \\
\hline 12.6560 & 16050 & 0.3072 & -18164 & 0.3139 & 0.6259 & -6757 \\
\hline 13.4374 & 16223 & 0.3204 & -17991 & 0.3270 & 0.6140 & -6970 \\
\hline 14.2431 & 16414 & 0.3334 & -17800 & 0.3399 & 0.6022 & -7178 \\
\hline 15.0654 & 16369 & 0.3463 & -17844 & 0.3526 & 0.5906 & -7384 \\
\hline 15.9136 & 16363 & 0.3589 & -17851 & 0.3652 & 0.5791 & -7587 \\
\hline \multicolumn{7}{|c|}{$i=\mathrm{Ni}$; starting amounts: $n_{\mathrm{Sn}}=25.2818 \times 10^{-3} \mathrm{~mol} ; n_{\mathrm{Zn}}=2.4438 \times 10^{-3} \mathrm{~mol}$} \\
\hline 0 & $\ldots$ & 0 & $\ldots$ & 0 & 0.9119 & 687 \\
\hline 0.4330 & -25481 & 0.0077 & -59751 & 0.0154 & 0.8978 & -242 \\
\hline 0.8784 & -24916 & 0.0230 & -59186 & 0.0307 & 0.8839 & -1160 \\
\hline 1.8115 & -14379 & 0.0460 & -48649 & 0.0613 & 0.8559 & -2660 \\
\hline 2.3156 & -2355 & 0.0692 & -36625 & 0.0771 & 0.8416 & -3230 \\
\hline 2.8352 & 4419 & 0.0849 & -29851 & 0.0928 & 0.8273 & -3683 \\
\hline 3.3701 & 15772 & 0.1006 & -18497 & 0.1084 & 0.8130 & -3938 \\
\hline 3.9239 & 10040 & 0.1162 & -24229 & 0.1240 & 0.7988 & -4293 \\
\hline 5.0895 & 15527 & 0.1395 & -18742 & 0.1551 & 0.7704 & -4806 \\
\hline 5.6884 & 15422 & 0.1627 & -18848 & 0.1702 & 0.7566 & -5058 \\
\hline 6.2987 & 16705 & 0.1777 & -17564 & 0.1851 & 0.7431 & -5282 \\
\hline 6.9339 & 16576 & 0.1926 & -17694 & 0.2001 & 0.7294 & -5509 \\
\hline
\end{tabular}


Table 2 continued

\begin{tabular}{|c|c|c|c|c|c|c|}
\hline \multirow{2}{*}{$\begin{array}{l}\text { Dropped mole } \\
n_{\mathrm{i}}, 10^{-3} \mathrm{~mol}\end{array}$} & \multirow{2}{*}{$\begin{array}{c}\text { Drop enthalpy } \\
\Delta H_{\text {Signal }}, \mathrm{J} \mathrm{mol}^{-1}\end{array}$} & \multicolumn{2}{|c|}{ Partial enthalpy } & \multicolumn{3}{|c|}{ Integral enthalpy(a) } \\
\hline & & $x_{i}(\mathbf{b})$ & $\Delta \overline{\boldsymbol{H}_{i}}, \mathbf{J} \mathrm{mol}^{-1}$ & $x_{\mathrm{Ni}}$ & $x_{\mathrm{Sn}}$ & $\Delta_{\mathrm{Mix}} \boldsymbol{H}, \mathrm{J} \mathrm{mol}^{-1}$ \\
\hline 7.5933 & 16046 & 0.2075 & -18223 & 0.2150 & 0.7158 & -5747 \\
\hline 8.2679 & 17026 & 0.2223 & -17244 & 0.2297 & 0.7024 & -5962 \\
\hline 8.9444 & 16662 & 0.2368 & -17607 & 0.2439 & 0.6894 & -6177 \\
\hline 9.6405 & 16232 & 0.2510 & -18037 & 0.2580 & 0.6766 & -6398 \\
\hline 10.3527 & 16463 & 0.2649 & -17806 & 0.2719 & 0.6639 & -6611 \\
\hline 11.0981 & 16721 & 0.2789 & -17548 & 0.2859 & 0.6512 & -6821 \\
\hline 11.8519 & 16640 & 0.2927 & -17630 & 0.2995 & 0.6388 & -7027 \\
\hline 12.6078 & 16581 & 0.3060 & -17689 & 0.3126 & 0.6268 & -7227 \\
\hline 13.3807 & 16234 & 0.3191 & -18036 & 0.3255 & 0.6150 & -7430 \\
\hline 14.1806 & 16382 & 0.3320 & -17887 & 0.3384 & 0.6033 & -7630 \\
\hline 15.0078 & 16453 & 0.3448 & -17817 & 0.3512 & 0.5916 & -7827 \\
\hline 15.8386 & 16186 & 0.3574 & -18083 & 0.3636 & 0.5803 & -8023 \\
\hline \multicolumn{7}{|c|}{ Sect. $D: x_{\mathrm{Sn}} / x_{\mathrm{Zn}} \approx 4: 1 ; i=\mathrm{Ni}$; starting amounts: $n_{\mathrm{Sn}}=25.2954 \times 10^{-3} \mathrm{~mol} ; n_{\mathrm{Zn}}=5.5993 \times 10^{-3} \mathrm{~mol}$} \\
\hline 0 & $\ldots$ & 0 & $\ldots$ & 0 & 0.8188 & 1409 \\
\hline 0.4356 & -27712 & 0.0070 & -61936 & 0.0139 & 0.8074 & 528 \\
\hline 0.8942 & -27644 & 0.0210 & -61867 & 0.0281 & 0.7957 & -372 \\
\hline 2.3401 & -1591 & 0.0493 & -35814 & 0.0704 & 0.7611 & -1914 \\
\hline 2.8627 & 14270 & 0.0776 & -19953 & 0.0848 & 0.7493 & -2193 \\
\hline 3.3958 & 17296 & 0.0919 & -16928 & 0.0990 & 0.7377 & -2422 \\
\hline 3.9385 & 16899 & 0.1060 & -17325 & 0.1131 & 0.7262 & -2655 \\
\hline 4.5007 & 14899 & 0.1201 & -19325 & 0.1272 & 0.7147 & -2919 \\
\hline 5.0752 & 17149 & 0.1341 & -17074 & 0.1411 & 0.7032 & -3145 \\
\hline 5.6794 & 16141 & 0.1482 & -18083 & 0.1553 & 0.6916 & -3392 \\
\hline 6.2922 & 16243 & 0.1622 & -17980 & 0.1692 & 0.6802 & -3633 \\
\hline 6.9180 & 16587 & 0.1761 & -17636 & 0.1830 & 0.6690 & -3864 \\
\hline 7.5767 & 16342 & 0.1899 & -17881 & 0.1969 & 0.6575 & -4104 \\
\hline 8.2482 & 15991 & 0.2038 & -18232 & 0.2107 & 0.6462 & -4347 \\
\hline 8.9409 & 16355 & 0.2176 & -17869 & 0.2244 & 0.6350 & -4582 \\
\hline 9.6419 & 16651 & 0.2312 & -17572 & 0.2379 & 0.6240 & -4806 \\
\hline 10.3848 & 16676 & 0.2447 & -17547 & 0.2516 & 0.6128 & -5036 \\
\hline 11.1370 & 16680 & 0.2583 & -17543 & 0.2650 & 0.6018 & -5260 \\
\hline 11.9000 & 16546 & 0.2715 & -17678 & 0.2781 & 0.5911 & -5481 \\
\hline 12.6854 & 16366 & 0.2846 & -17857 & 0.2911 & 0.5804 & -5704 \\
\hline 13.4884 & 16751 & 0.2975 & -17472 & 0.3039 & 0.5699 & -5917 \\
\hline 14.3170 & 16507 & 0.3103 & -17716 & 0.3167 & 0.5595 & -6133 \\
\hline 15.1782 & 16826 & 0.3231 & -17397 & 0.3294 & 0.5490 & -6344 \\
\hline \multicolumn{7}{|c|}{$i=\mathrm{Ni}$; starting amounts: $n_{\mathrm{Sn}}=25.2995 \times 10^{-3} \mathrm{~mol} ; n_{\mathrm{Zn}}=5.5168 \times 10^{-3} \mathrm{~mol}$} \\
\hline 0 & $\ldots$ & 0 & $\ldots$ & 0 & 0.8210 & 1392 \\
\hline 0.4360 & -29183 & 0.0070 & -63435 & 0.0140 & 0.8095 & 488 \\
\hline 0.8924 & -28183 & 0.0210 & -62434 & 0.0281 & 0.7979 & -418 \\
\hline 1.3610 & -27581 & 0.0352 & -61833 & 0.0423 & 0.7863 & -1312 \\
\hline 1.8353 & -15096 & 0.0493 & -49347 & 0.0562 & 0.7748 & -2010 \\
\hline 2.3273 & -10944 & 0.0632 & -45196 & 0.0702 & 0.7633 & -2651 \\
\hline 2.8471 & 14858 & 0.0774 & -19394 & 0.0846 & 0.7515 & -2910 \\
\hline 3.3788 & 16836 & 0.0917 & -17416 & 0.0988 & 0.7399 & -3135 \\
\hline 3.9352 & 16461 & 0.1060 & -17790 & 0.1132 & 0.7280 & -3370 \\
\hline 4.5002 & 16638 & 0.1203 & -17614 & 0.1274 & 0.7164 & -3598 \\
\hline 5.0793 & 16517 & 0.1345 & -17734 & 0.1415 & 0.7048 & -3826 \\
\hline 5.6901 & 15904 & 0.1487 & -18347 & 0.1559 & 0.6930 & -4069 \\
\hline 6.3075 & 16388 & 0.1629 & -17864 & 0.1699 & 0.6815 & -4298 \\
\hline 6.9417 & 16140 & 0.1769 & -18112 & 0.1838 & 0.6700 & -4530 \\
\hline 7.5937 & 16510 & 0.1908 & -17741 & 0.1977 & 0.6587 & -4755 \\
\hline 8.2566 & 16364 & 0.2045 & -17888 & 0.2113 & 0.6475 & -4977 \\
\hline 8.9334 & 15615 & 0.2180 & -18637 & 0.2247 & 0.6365 & -5210 \\
\hline
\end{tabular}


Table 2 continued

\begin{tabular}{|c|c|c|c|c|c|c|}
\hline \multirow{2}{*}{$\begin{array}{l}\text { Dropped mole } \\
n_{\mathrm{i}}, 10^{-3} \mathrm{~mol}\end{array}$} & \multirow{2}{*}{$\begin{array}{c}\text { Drop enthalpy } \\
\Delta H_{\text {Signal }}, \mathrm{J} \mathrm{mol}^{-1}\end{array}$} & \multicolumn{2}{|c|}{ Partial enthalpy } & \multicolumn{3}{|c|}{ Integral enthalpy(a) } \\
\hline & & $x_{i}(\mathbf{b})$ & $\Delta \overline{\boldsymbol{H}_{i}}, \mathrm{~J} \mathrm{~mol}^{-1}$ & $x_{\mathrm{Ni}}$ & $x_{\mathrm{Sn}}$ & $\Delta_{\mathrm{Mix}} \boldsymbol{H}, \mathrm{J} \mathrm{mol}^{-1}$ \\
\hline 9.6490 & 16360 & 0.2316 & -17892 & 0.2385 & 0.6252 & -5434 \\
\hline 10.3765 & 16451 & 0.2452 & -17801 & 0.2519 & 0.6142 & -5653 \\
\hline 11.1069 & 17586 & 0.2584 & -16666 & 0.2649 & 0.6035 & -5845 \\
\hline 11.8574 & 16722 & 0.2714 & -17530 & 0.2779 & 0.5929 & -6050 \\
\hline 12.6251 & 17009 & 0.2842 & -17242 & 0.2906 & 0.5824 & -6248 \\
\hline 13.4052 & 16959 & 0.2969 & -17293 & 0.3031 & 0.5721 & -6443 \\
\hline 14.1947 & 16430 & 0.3092 & -17822 & 0.3154 & 0.5621 & -6642 \\
\hline 15.0158 & 16178 & 0.3215 & -18073 & 0.3276 & 0.5520 & -6847 \\
\hline 15.8574 & 16070 & 0.3337 & -18182 & 0.3398 & 0.5421 & -7051 \\
\hline
\end{tabular}

(a) Per mole of mixture

(b) Average value before and after the drop

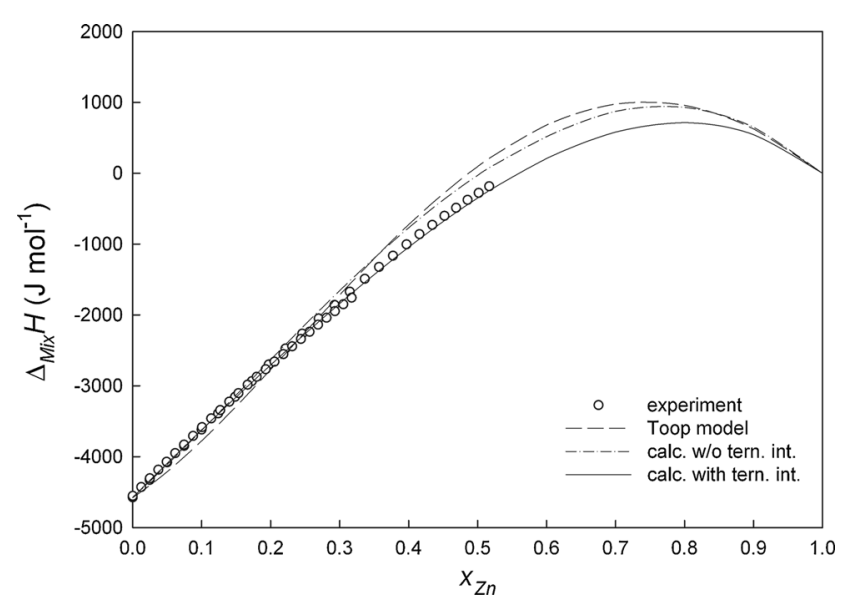

Fig. 2 Integral molar enthalpies of mixing of liquid Ni-Sn-Zn alloys along the section $x_{\mathrm{Ni}} / x_{\mathrm{Sn}} \approx 1: 9$ at $1073 \mathrm{~K}$; reference states: pure liquid metals

\section{Results and Discussion}

\subsection{Experimental Results}

According to the phase equilibria at $1073 \mathrm{~K},{ }^{[35]}$ the experimental temperature for $(\mathrm{Ni}-\mathrm{Sn})+\mathrm{Zn}$ alloys along the sections $\mathrm{A}\left(x_{\mathrm{Ni}} / x_{\mathrm{Sn}} \approx 1: 9\right)$ and $\mathrm{B}\left(x_{\mathrm{Ni}} / x_{\mathrm{Sn}} \approx 1: 6\right)$ was high enough to obtain completely liquid alloys over the entire investigated concentration range; see dashed-points line in Fig. 1. In contrary, the clear kinks in the enthalpy curves for $(\mathrm{Sn}-\mathrm{Zn})+\mathrm{Ni}$ alloys along the cross sections $\mathrm{C}\left(x_{\mathrm{Sn}}\right)$ $\left.x_{\mathrm{Zn}}=9: 1\right)$ and $\mathrm{D}\left(x_{\mathrm{Sn}} / x_{\mathrm{Zn}} \approx 4: 1\right)$ shown in Fig. 4 and 5 indicate formation of a solid phase and denote the liquidus limit at $873 \mathrm{~K}$. The corresponding points are in reasonable agreement with the estimated liquidus line at $873 \mathrm{~K}$ given by Yuan et al. ${ }^{[3]}$ which is shown as a dashed line in Fig. 1.

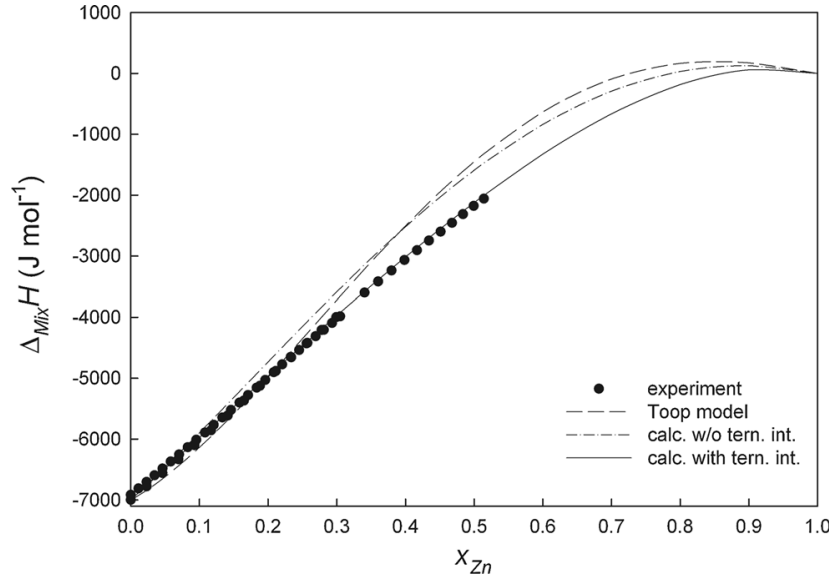

Fig. 3 Integral molar enthalpies of mixing of liquid Ni-Sn-Zn alloys along the section $x_{\mathrm{Ni}} / x_{\mathrm{Sn}} \approx 1: 6$ at $1073 \mathrm{~K}$; reference states: pure liquid metals

Accordingly, the values within the italicized values in Table 2 are for alloys beyond the liquidus limit.

The obtained enthalpies of mixing are exothermic along all sections, indicating the preferred interactions between unlike kinds of atoms. It should be noted that the enthalpy of mixing data for both $(\mathrm{Sn}-\mathrm{Zn})+\mathrm{Ni}$ alloys are practically identical in the concentration range after formation of a solid phase. This may be explained by formation of the same phase in both cases.

\subsection{Ternary Modeling}

The interaction parameters of the binary systems were taken directly from the literature ${ }^{[24,32,40]}$ and are listed in Table 3 . The enthalpy of mixing for the ternary system was treated by a least-squares fit using the following Redlich- 


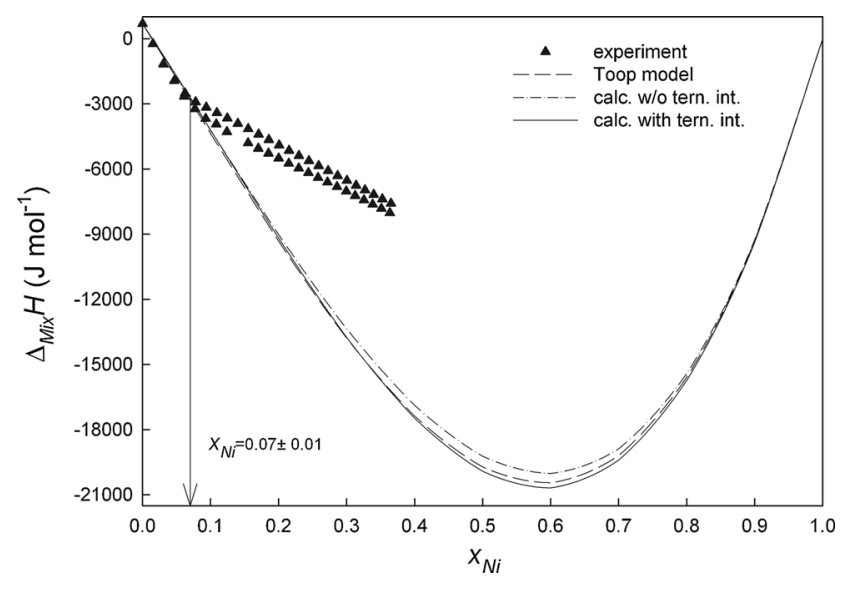

Fig. 4 Integral molar enthalpies of mixing of liquid Ni-Sn-Zn alloys along the section $x_{\mathrm{Sn}} / x_{\mathrm{Zn}} \approx 9: 1$ at $873 \mathrm{~K}$; reference states: pure liquid metals

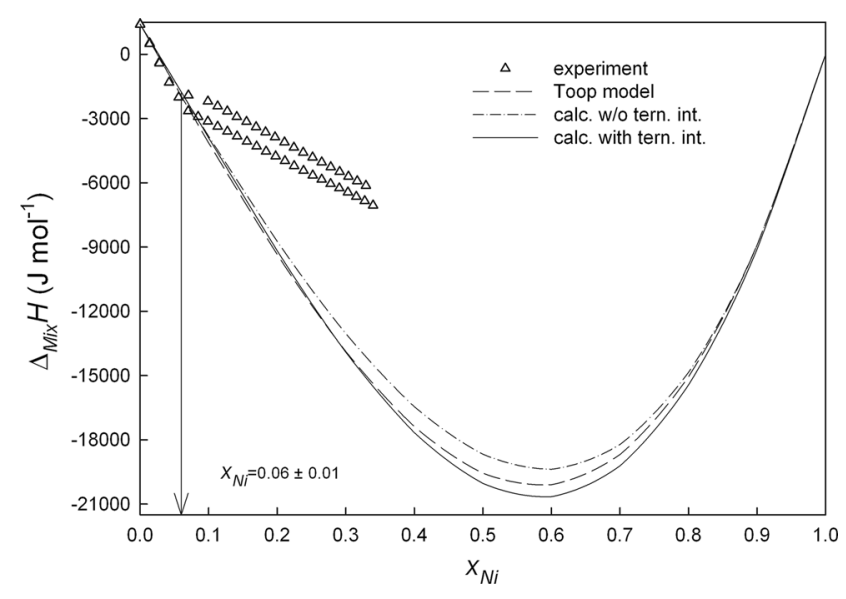

Fig. 5 Integral molar enthalpies of mixing of liquid Ni-Sn-Zn alloys along the section $x_{\mathrm{Sn}} / x_{\mathrm{Zn}} \approx 4: 1$ at $873 \mathrm{~K}$; reference states: pure liquid metals

Kister-Muggianu polynomial, ${ }^{[9]}$ which takes into account additional ternary interactions:

$$
\begin{aligned}
\Delta_{\text {Mix }} H= & \sum_{i} \sum_{j>i}\left[x_{i} x_{j} \sum_{v}{ }^{v} L_{i: j}\left(x_{i}-x_{j}\right)^{v}\right] \\
& +x_{i} x_{j} x_{k}\left({ }^{0} M_{i: j: k} x_{i}+{ }^{1} M_{i: j: k} x_{j}+{ }^{2} M_{i: j: k} x_{k}\right),
\end{aligned}
$$

where $i, j, k$ are equal to $1,2,3$ for the elements $\mathrm{Ni}, \mathrm{Sn}$ and $\mathrm{Zn}$ respectively; ${ }^{v} L_{i: j}(v=0,1,2, \ldots)$ are the interaction parameters of the three binary systems; ${ }^{v} M_{i: j: k}(v=0,1,2)$ are three ternary interaction parameters; $x_{i}, x_{j}, x_{k}$ are the corresponding mole fractions. The enthalpy of mixing is temperature independent for the two binary systems Ni-Zn and $\mathrm{Sn}-\mathrm{Zn}$, and it shows small temperature dependence for the Ni-Sn system. Therefore, any possible temperature dependence of $\Delta_{\mathrm{Mix}} H$ in the ternary Ni-Sn-Zn system was
Table 3 Binary and ternary interaction parameters for Ni-Sn-Zn

\begin{tabular}{lcl}
\hline System & Interaction parameters, $\mathbf{J ~ m o l}^{\mathbf{1}}$ & Reference \\
\hline Ni-Sn & ${ }^{0} \mathrm{~L}=-80659+183 * T$ & \\
& ${ }^{1} \mathrm{~L}=-24617-953 * T$ \\
Ni-Zn & ${ }^{0} \mathrm{~L}=-50722$ & \\
& ${ }^{1} \mathrm{~L}=8436$ & \\
Sn-Zn & ${ }^{2} \mathrm{~L}=-25136$ \\
& ${ }^{0} \mathrm{~L}=12728$ & \\
Ni-Sn-Zn & ${ }^{1} \mathrm{~L}=-5074$ & \\
& ${ }^{0} \mathrm{M}=-156468$ & \\
& ${ }^{1} \mathrm{M}=26414$ & \\
& ${ }^{2} \mathrm{M}=-64909$ & Present work
\end{tabular}

Temperatures $(T)$ in kelvin

neglected in the present evaluation. The parameters ${ }^{v} M_{i: j: k}$, obtained from the experimental enthalpy of mixing data, represent the additional contribution due to ternary interactions (Table 3). The difference between experimental and calculated enthalpy of mixing data is not more than $\pm 250 \mathrm{~J} \mathrm{~mol}^{-1}$ which is within the limits of the experimental errors. This can be seen from Fig. 2, 3, 4, and 5 where full lines refer to calculated values with ternary interaction, dashed lines to those without.

As an alternative, the so-called Toop model ${ }^{[10]}$ was used to calculate the ternary enthalpy values. This model uses an asymmetric extrapolation to predict ternary thermodynamic quantities based on binary data. The corresponding equation is:

$$
\begin{aligned}
\Delta_{\mathrm{Mix}} H= & \frac{x_{j}}{1-x i} \Delta_{\mathrm{Mix}} H_{i, j}\left(x_{i}, 1-x_{i}\right)+\frac{x_{k}}{1-x_{i}} \Delta_{\mathrm{Mix}} H_{i, k}\left(x_{i}, 1-x_{i}\right) \\
& +\left(x_{j}+x_{k}\right)^{2} \Delta_{\mathrm{Mix}} H_{j, k}\left(\frac{x_{j}}{x_{j}+x_{k}}, \frac{x_{k}}{x_{j}+x_{k}}\right),
\end{aligned}
$$

where $\Delta_{\mathrm{Mix}} H_{i, j}, \Delta_{\mathrm{Mix}} H_{i, k}$, and $\Delta_{\mathrm{Mix}} H_{j, k}$, are the enthalpies of mixing for liquid Ni-Sn, Ni-Zn and $\mathrm{Sn}-\mathrm{Zn}$ alloys, respectively. The enthalpies of mixing values of binary subsystems were calculated by a Redlich-Kister polynomial based on interaction parameters from the literature given in Table 3.

A comparison of the experimental enthalpy of mixing with the calculated data along all investigated cross sections is shown in Fig. 2, 3, 4, and 5. It can be seen that the calculated curves based on the Toop model are in good agreement with our fitting without ternary interaction terms and differ from the experimental data by less than $400 \mathrm{~J} \mathrm{~mol}^{-1}$ except for the section $x_{\mathrm{Ni}} / x_{\mathrm{Sn}} \approx 1: 9$ where the deviation is higher. This comparatively small improvement of the fits adding ternary interaction terms, however, is not a proof for the existence of real ternary interaction in the liquid phase. Both, the Muggianu- and the Toop-model for the extrapolation of binary enthalpy data into the ternary are of limiting significance. Thus the ternary terms could also compensate shortcomings of the binary extrapolations models 


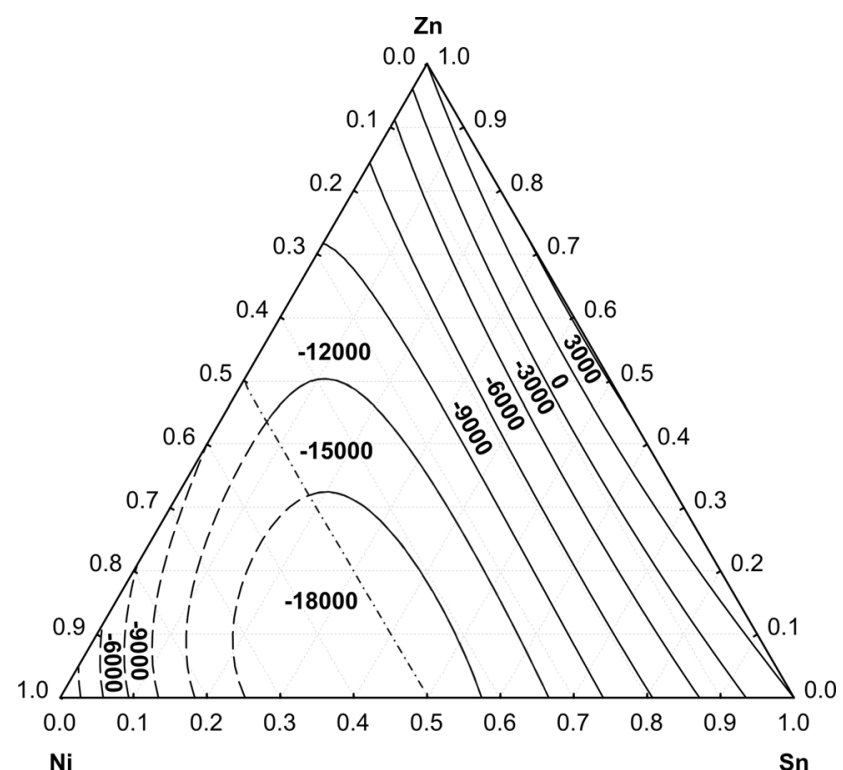

Fig. 6 Isoenthalpy curves of liquid Ni-Sn-Zn alloys valid for the temperature range $873-1073 \mathrm{~K}$; reference states: pure liquid metals, numbers given in $\mathrm{J} \mathrm{mol}^{-1}$

Finally, an iso-enthalpy plot is presented in Fig. 6. The values are exothermic in most of the ternary composition range, except close to the binary $\mathrm{Sn}-\mathrm{Zn}$ system. The minimum values are actually in the binary Ni-Sn system. All data beyond the liquidus limit are considered as enthalpy of mixing of the metastable liquid.

\section{Conclusions}

Enthalpies of mixing in the liquid Ni-Sn-Zn system were measured along four sections using a high temperature Calvet microcalorimeter. For two sections $x_{\mathrm{Ni}} / x_{\mathrm{Sn}} \approx 1: 9, x_{\mathrm{Ni}} /$ $x_{\mathrm{Sn}} \approx 1: 6$ were measured at $1073 \mathrm{~K}$, for the other two sections, i.e. $x_{\mathrm{Sn}} / x_{\mathrm{Zn}} \approx 9: 1, x_{\mathrm{Sn}} / x_{\mathrm{Zn}} \approx 4: 1$, experiments were performed at $873 \mathrm{~K}$. A comparison of experimental and calculated enthalpy of mixing values based on RedlichKister-Muggianu data fits and on the Toop extrapolation model shows good agreement.

Based on the experimental data three ternary interaction parameters ${ }^{v} M_{i: j: k}$ were obtained according to the RedlichKister-Muggianu polynomial. These data could be used in a standard CALPHAD procedure for the assessment of the equilibrium phase diagram.

\section{Acknowledgments}

This work was performed in the framework of the European Concerted Research Action COST MP0602 project "Advanced Solder Materials for High Temperature Application". Financial support by the Austrian Science Fund (FWF) under Project No. P21507 is gratefully acknowledged. Yu.P. was supported through a Short Term
Scientific Mission within COST MP0602. A.Ya. was supported by a Lise Meitner Fellowship of the Austrian Science Fund (FWF, Project No. M1324).

\section{Open Access}

This article is distributed under the terms of the Creative Commons Attribution License which permits any use, distribution, and reproduction in any medium, provided the original author(s) and the source are credited.

\section{References}

1. K.J. Puttlitz and K.A. Stalter, Handbook of Lead-Free Solder Technology for Microelectronic Assemblies, Marcel Dekker Inc., New York, 2004

2. K.S. Kim, J.M. Yang, C.H. Yu, I.O. Jung, and H.H. Kim, Analysis on Interfacial Reactions Between Sn-Zn Solders and the Au/Ni Electrolytic-Plated Cu Pad, J. Alloys Compd., 2004, 379, p 314-318

3. I. Shohji, T. Nakamura, F. Mori, and S. Fujiuchi, Interface Reaction and Mechanical Properties of Lead-free Sn-Zn Alloy/ Cu Joints, Mater. Trans., 2002, 43, p 1797-1801

4. K. Suganuma and K.S. Kim, Sn-Zn Low Temperature Solder, J. Mater. Sci. Mater. Electron., 2007, 18, p 121-127

5. X.Q. Wei, H.Z. Huang, L. Zhou, M. Zhang, and X.D. Liu, On the Advantages of Using a Hypoeutectic Sn-Zn as Lead-Free Solder Material, Mater. Lett., 2007, 61, p 655-658

6. J.X. Jiang, J.E. Lee, K.S. Kim, and K. Suganuma, Oxidation Behavior of Sn-Zn Solders Under High-Temperature and High-Humidity Conditions, J. Alloys Compd., 2008, 462, p 244-251

7. W.J. Zhu, H.S. Liu, J.S. Wang, G. Ma, and Z.P. Jin, Interfacial Reactions Between Sn-Zn Alloys and Ni Substrates, J. Electron. Mater., 2010, 39, p 209-214

8. A.K. Gain, Y.C. Chan, and W.K.C. Yung, Effect of Nano Ni Additions on the Structure and Properties of Sn-9Zn and Sn$\mathrm{Zn}-3 \mathrm{Bi}$ Solders in $\mathrm{Au} / \mathrm{Ni} / \mathrm{Cu}$ Ball Grid Array Packages, Mater. Sci. Eng. B, 2009, 162, p 92-98

9. Y.M. Muggianu, M. Gambino, and J.P. Bros, Enthalpies of Formation of Liquid Alloys Bismuth-Gallium-Tin at $723 \mathrm{~K}$ - Choice of an Analytical Representation of Integral and Partial Thermodynamic Functions of Mixing for This Ternary-System, J. Chim. Phys., 1975, 72, p 83-88

10. G.W. Toop, Predicting Ternary Activities Using Binary Data, Trans. Met. Soc. AIME, 1965, 233, p 850-855

11. O.J. Kleppa, A Thermodynamic Study of Liquid Metallic Solutions. 6. Calorimetric Investigations of the Systems Bismuth-Lead, Cadmium-Lead, Cadmium-Tin and Tin-Zinc, J. Phys. Chem., 1955, 59, p 354-361

12. W. Oelsen, Zur Kalorimetrie Und Thermodynamik Der ZinnZink-Legierungen, Z. Metallkd., 1957, 48, p 1-8, in German

13. E. Schürmann and H. Träger, Die Empfindlichkeit und die Wiederholbarkeit von Messungen mit dem Kleinkalorimeter (The Sensitivity and Reproducibility of Measurements with Microcalorimeter), Arch. Eisenhüttenwes., 1961, 32, p 397408

14. Z. Moser and R.S. K. Rzyman, Calorimetric Studies on Zn-Sn Liquid Solutions, Bull. Pol. Acad. Sci. Tech. Sci., 1987, 35, p 461-464

15. M. Genot and R. Hagege, Etude Thermodynamique Du Systeme Etainzinc, Compt. Rend. Hebd. Acad. Sci., 1960, 251, p 2901-2903, in French 
16. W. Ptak, Thermodynamics of Liquid Zinc-Tin Solutions, Archiwum Hutnictwa, 1960, 5, p 649-650

17. K. Sano, K. Okajima, and S. Tatsuo, A Thermodynamic Study of the Liquid Ternary System Zinc-Cadmium and Zinc-Tin. III. The System Zinc-Cadmium-Tin, Mem. Fac. Eng. Nagoya Univ., 1953, 5, p 299-305

18. M. Fiorani and V. Valenti, Ricerche Termodinamiche su Sistemi Metallici - Nota III. Sistema Liquido Zonco-Stango, Gazz. Chim. Ital., 1955, 85, p 607-615, in Italian

19. E. Scheil and E.D. Muller, Dampfdruckmessungen an Flussigen Zink-Zinn-Legierungen, Z. Metallkd., 1962, 53, p 389395 , in German

20. K. Itagaki and A. Yazawa, Measurements of Thermo-Dynamic Quantities for Tin-Zinc and Indium-Antimony Alloys by Quantitative Thermal Analysis, J. Jpn. Inst. Met., 1975, 39, p 880-887

21. B.J. Lee, Thermodynamic Assessments of the Sn-Zn and InZn Binary Systems, CALPHAD, 1996, 20, p 471-480

22. R. Haddad, M. Gaune-Escard, J.P. Bros, A. RanningerHavlicek, E. Hayer, and K.L. Komarek, Thermodynamics of Nickel-Tin Liquid Alloys, J. Alloys Compd., 1997, 247, p 8292

23. R. Luck, J. Tomiska, and B. Predel, Calorimetric Determination of the Enthalpy of Mixing of Liquid Nickel-Tin Alloys as a Function of Temperature, Z. Metallkd., 1988, 79, p 345-349, in German

24. H. Flandorfer, C. Luef, and U. Saeed, On the Temperature Dependence of the Enthalpies of Mixing in Liquid Binary (Ag, Cu, Ni)-Sn Alloys, J. Non-Cryst. Solids, 2008, 354, p 29532972

25. P. Nash, H. Choo, and R.B. Schwarz, Thermodynamic Calculation of Phase Equilibria in the Ti-Co and Ni-Sn Systems, J. Mater. Sci., 1998, 33, p 4929-4936

26. G. Ghosh, Thermodynamic Modeling of the Nickel-Lead-Tin System, Metall. Mater. Trans. A, 1999, 30, p 1481-1494

27. H.S. Liu, J. Wang, and Z.P. Jin, Thermodynamic Optimization of the Ni-Sn Binary System, CALPHAD, 2004, 28, p 363-370

28. H. Hagiwara, S. Sugino, and K. Yamaguchi, Activity of Zinc in Liquid Nickel-Zinc Alloys, Bull. Univ. Osaka Prefect. Ser. A, 1977, 26, p 81-86
29. T.G. Chart, J.K. Critchle, and R. Williams, Thermodynamic Data for Nickel-Zinc Alloys, J. Inst. Met., 1968, 96, p 224

30. G.P. Vassilev, T. Gomez-Acebo, and J.C. Tedenac, Thermodynamic Optimization of the Ni-Zn System, J. Phase Equilib., 2000, 21, p 287-301

31. X.P. Su, N.Y. Tang, and J.M. Toguri, Thermodynamic Assessment of the Ni-Zn System, J. Phase Equilib., 2002, 23, p 140-148

32. J. Miettinen, Thermodynamic Description of the $\mathrm{Cu}-\mathrm{Ni}-\mathrm{Zn}$ System Above 600 C, CALPHAD, 2003, 27, p 263-274

33. P. Franke and D. Neuschuetz, Ni-Zn (Nickel-Zinc) Binary System, in Part 5: Binary Systems Supplement 1. LandoltBoernstein - Group IV Physical Chemistry, 2007, p 1-4

34. J. Chang, S.K. Seo, and H.M. Lee, Phase Equilibria in the Sn$\mathrm{Ni}-\mathrm{Zn}$ Ternary System: Isothermal Sections at $200^{\circ} \mathrm{C}, 500^{\circ} \mathrm{C}$, and $800^{\circ} \mathrm{C}, J$. Electron. Mater, 2010, 39, p 2643-2652

35. C. Schmetterer, D. Rajamohan, H. Ipser, and H. Flandorfer, The High-Temperature Phase Equilibria of the Ni-Sn-Zn System: Isothermal Sections, Intermetallics, 2011, 19, p 1489-1501

36. V. Gandova, J. Romanowska, and G. Vassilev, Comparison Between Sn-Ni-Zn Liquid Phase Thermodynamic Assessments Performed by the CALPHAD Method and by Geometrical Models, RMZ M. \& G., 2010, 57, p 441-452

37. Y. Yuan, S. Delsante, D.J. Li, and G. Borzone, The Isothermal Section of the Ni-Sn-Zn Phase Diagram at $873 \mathrm{~K}$, Intermetallics, 2011, 19, p 1646-1650

38. H. Flandorfer, F. Gehringer, and E. Hayer, Individual Solutions for Control and Data Acquisition with the PC, Thermochim. Acta, 2002, 382, p 77-87

39. A.T. Dinsdale, SGTE Data for Pure Elements, CALPHAD, 1991, 15, p 317-425

40. M. Rechchach, A. Sabbar, H. Flandorfer, and H. Ipser, Enthalpies of Mixing of Liquid In-Sn and In-Sn-Zn Alloys, Thermochim. Acta, 2010, 502, p 66-72

41. V. Gandova, D. Soares, K. Lilova, J.-C. Tedenac, and G.P. Vassilev, Phase Equilibria in the Sn-Zn-Ni System, Int. J. Mater. Res., 2011, 102, p 257-268

42. V.D. Gandova, P. Broz, J. Buršík, and G.P. Vassilev, Thermochemical and Phase Diagram Studies of the Sn-Zn-Ni System, Thermochim. Acta, 2011, 524, p 47-55 